\title{
Erratum to: Trends and determinants of inequities in childhood stunting in Bangladesh from 1996/7 to 2014
}

\author{
Atonu Rabbani ${ }^{1}$, Akib Khan ${ }^{2 *}$, Sifat Yusuf ${ }^{3}$ and Alayne Adams ${ }^{3}$
}

\section{Erratum}

Unfortunately, after publication of this article [1], it was noticed that Tables 3 and 4 were inadvertently swapped during the production process. The tables with their correct table citation can be seen below and the original article has been update to reflect this.

\footnotetext{
Author details

${ }^{1}$ Department of Economics, University of Dhaka, Dhaka, Bangladesh. ${ }^{2}$ James P. Grant School of Public Health, BRAC University, Dhaka, Bangladesh. ${ }^{3}$ Health Systems and Population Sciences Division, International Center for Diarrheal

Disease Research (icddr,b), Dhaka, Bangladesh.
}

Received: 7 December 2016 Accepted: 8 December 2016

Published online: 04 January 2017

\section{Reference}

1. Rabbani A, Khan A, Yusuf S, Adams A. Trends and determinants of inequities

in childhood stunting in Bangladesh from 1996/7 to 2014. Int J Equity

Health. 2016;15:186. http://doi.org/10.1186/s12939-016-0477-7.

\footnotetext{
* Correspondence: akib.econdu@gmail.com

${ }^{2}$ James P. Grant School of Public Health, BRAC University, Dhaka, Bangladesh
} 
Table 3 Decomposition of the concentration index for stunting (1996/97 \& 2014)

\begin{tabular}{|c|c|c|c|c|c|c|c|c|c|c|c|}
\hline \multirow{3}{*}{$\frac{\text { Variables }}{\text { Child's age }}$} & \multicolumn{4}{|c|}{ Coefficients \& $p$-values } & \multicolumn{2}{|c|}{ Elasticities } & \multicolumn{2}{|c|}{ Concentration index (C) } & \multicolumn{3}{|c|}{ Contributions to C (\%) } \\
\hline & \multicolumn{2}{|c|}{ 1996/97 } & \multicolumn{2}{|l|}{2014} & \multirow{2}{*}{$\begin{array}{l}1996 / 97 \\
1.30\end{array}$} & \multirow{2}{*}{$\begin{array}{l}2014 \\
2.48\end{array}$} & \multirow{2}{*}{$\begin{array}{l}1996 / 97 \\
-0.006\end{array}$} & \multirow{2}{*}{$\begin{array}{l}2014 \\
-0.009\end{array}$} & \multirow{2}{*}{$\begin{array}{l}1996 / 97 \\
9.58\end{array}$} & \multirow{2}{*}{$\frac{2014}{13.30}$} & \multirow{2}{*}{$\frac{\text { Change }}{3.71}$} \\
\hline & 0.03 & 0.00 & 0.03 & 0.00 & & & & & & & \\
\hline Child's age squared & 0.00 & 0.00 & 0.00 & 0.00 & -0.68 & -1.82 & -0.005 & -0.016 & -4.82 & -17.92 & -13.10 \\
\hline Birth Order & 0.00 & 0.35 & -0.01 & 0.28 & 0.02 & -0.04 & -0.042 & -0.083 & 1.02 & -1.89 & -2.90 \\
\hline Antenatal visit to doctor & -0.05 & 0.02 & -0.03 & 0.23 & -0.02 & -0.04 & 0.363 & 0.199 & 8.14 & 5.25 & -2.89 \\
\hline Delivery at health facility & -0.05 & 0.16 & -0.04 & 0.05 & 0.00 & -0.04 & 0.638 & 0.302 & 3.09 & 7.02 & 3.93 \\
\hline Early initiation of breastfeeding & -0.07 & 0.00 & 0.02 & 0.19 & -0.02 & 0.03 & 0.062 & -0.055 & 1.67 & 1.15 & -0.52 \\
\hline Maternal Schooling (years) & -0.01 & 0.00 & -0.01 & 0.00 & -0.04 & -0.17 & 0.409 & 0.188 & 22.81 & 19.85 & -2.97 \\
\hline Paternal Schooling (years) & -0.01 & 0.00 & 0.00 & 0.35 & -0.05 & -0.03 & 0.343 & 0.260 & 23.53 & 5.34 & -18.19 \\
\hline Maternal CED (Chronic Energy Deficiency) & 0.02 & 0.27 & 0.03 & 0.10 & 0.02 & 0.02 & -0.101 & -0.246 & 2.10 & 3.11 & 1.02 \\
\hline Maternal short stature $(<145 \mathrm{~cm})$ & 0.17 & 0.00 & 0.19 & 0.00 & 0.05 & 0.07 & -0.043 & -0.149 & 2.79 & 6.04 & 3.25 \\
\hline Wealth Index & -0.04 & 0.00 & -0.03 & 0.03 & -0.29 & -0.41 & 0.074 & 0.105 & 27.19 & 26.47 & -0.72 \\
\hline Total & & & & & & & & & 97.11 & 67.72 & -29.38 \\
\hline
\end{tabular}

Note: $N=3,288$ for $1996 / 97$ and 3,884 for 2014. All regressions are probit. Coefficients are average marginal effects. Standard errors used take into account sampling weights. In addition to the variables reported, all regressions control for division-specific fixed effects. Antenatal visit to doctor, delivery at health facility, early breastfeeding, and maternal CED and short stature are all dummy variables

Table 4 Oaxaca decomposition for changes in stunting inequality between 1996/97 \& 2014

\begin{tabular}{|c|c|c|c|c|c|c|}
\hline \multirow[b]{3}{*}{$\underline{\text { Variables }}$} & \multicolumn{4}{|c|}{$\begin{array}{l}\text { E: Elasticity C: Conc. Index } \\
\text { t:2014 (t-1): 1996/97 }\end{array}$} & \multirow[b]{3}{*}{ Total } & \multirow[b]{3}{*}{$\%$} \\
\hline & \multicolumn{2}{|l|}{ Variation (1) } & \multicolumn{2}{|l|}{ Variation (2) } & & \\
\hline & $E_{t}\left(C_{t}-C_{t-1}\right)$ & $C_{t-1}\left(E_{t}-E_{t-1}\right)$ & $\mathrm{E}_{\mathrm{t}-1}\left(\mathrm{C}_{\mathrm{t}}-\mathrm{C}_{\mathrm{t}-1}\right)$ & $C_{t}\left(E_{t}-E_{t-1}\right)$ & & \\
\hline Child's age & -0.007 & -0.007 & -0.004 & -0.010 & -0.014 & 16.66 \\
\hline Child's age squared & 0.019 & 0.006 & 0.007 & 0.018 & 0.025 & -29.79 \\
\hline Birth Order & 0.002 & 0.002 & -0.001 & 0.005 & 0.004 & -4.52 \\
\hline Antenatal visit to doctor & 0.007 & -0.009 & 0.003 & -0.005 & -0.002 & 2.63 \\
\hline Delivery at health facility & 0.013 & -0.022 & 0.001 & -0.010 & -0.009 & 10.58 \\
\hline Early initiation of breastfeeding & -0.004 & 0.003 & 0.002 & -0.003 & -0.001 & 0.68 \\
\hline Maternal Schooling (years) & 0.038 & -0.052 & 0.010 & -0.024 & -0.015 & 17.16 \\
\hline Paternal Schooling (years) & 0.003 & 0.007 & 0.004 & 0.005 & 0.009 & -11.13 \\
\hline Maternal CED (Chronic Energy Deficiency) & -0.003 & 0.000 & -0.002 & -0.001 & -0.003 & 4.03 \\
\hline Maternal short stature $(<145 \mathrm{~cm})$ & -0.007 & -0.001 & -0.005 & -0.002 & -0.008 & 8.98 \\
\hline Wealth Index & -0.013 & -0.009 & -0.009 & -0.013 & -0.022 & 25.82 \\
\hline Residual & & & & & -0.050 & 58.89 \\
\hline Total & 0.047 & -0.082 & 0.006 & -0.041 & -0.085 & \\
\hline
\end{tabular}

Note: Variation 1 (2) uses $E_{t}\left(E_{t-1}\right)$ and $C_{t-1}\left(C_{t}\right)$ to weight changes in $C$ and $E$ respectively. See Wagstaff et al. (2003) for details 